\title{
Enhanced recognition of words previously presented in a task with nonfocal prospective memory requirements
}

\author{
Shayne Loft • Michael S. Humphreys
}

Published online: 31 July 2012

(C) Psychonomic Society, Inc. 2012

\begin{abstract}
Remembering to perform deferred actions when events are encountered in the future is referred to as eventbased prospective memory. Individuals can be slower to respond to ongoing tasks when they have prospective memory task requirements. These costs are interpreted as evidence for cognitive control processes allocated to the prospective memory task, but we know little about these processes. In the present article, the recognition of nontargets previously presented in an ongoing task with prospective memory task requirements provided evidence for the differential processing of individual ongoing task items. Participants performed a lexical decision task, where some participants were required to make an alternative prospective memory response either to a specific word (focal) or to exemplars of a category (nonfocal). Participants were slower to respond to the ongoing task in the nonfocal conditions than in the control condition (costs), regardless of whether or not prospective memory task importance was emphasized. Participants were also slower to respond to the ongoing task in the focal conditions than in the control condition, but only when prospective memory task importance was emphasized. This task was followed by a surprise recognition memory test in which nontarget words from the lexical decision task were intermixed with new words. Focal conditions, but not nonfocal conditions, showed better discrimination on the recognition task, as compared with the control condition. Participants in nonfocal conditions mapped the semantic features of the ongoing task letter strings onto the semantic features of their prospective memory category, and
\end{abstract}

S. Loft $(\bowtie)$

School of Psychology, The University of Western Australia,

Crawley, WA 6009, Australia

e-mail: Shayne.Loft@uwa.edu.au

M. S. Humphreys

The University of Queensland,

Brisbane, Australia this elaboration in the processing of individual nontargets increased incidental learning and produced the recognition benefit.

Keywords Prospective memory · Ongoing task costs · Recognition $\cdot$ Incidental learning

Many activities in our personal and professional lives depend on our ability to remember to perform deferred intended actions when certain events or stimuli are encountered. This type of task is referred to as event-based prospective memory (PM). The most widely used event-based PM paradigm, introduced by Einstein and McDaniel (1990), requires participants to remember to perform an atypical task action (e.g., press the F1 key) when presented with a target event (e.g., a particular syllable) while engaged in an ongoing task (e.g., categorization). Much of the PM literature has been concerned with delineating the cognitive mechanisms by which individuals remember to respond to targets with the PM response.

Numerous studies have demonstrated that response times to ongoing tasks on nontarget trials are slowed when individuals need to remember to perform PM tasks, an effect referred to as the costs to ongoing tasks (for a review, see Smith, Hunt, McVay, \& McConnell, 2007). Costs have been interpreted as evidence for the allocation of some form of attentional capacity to the PM task and have formed the primary dependent measure for determining the resource requirements of PM (Einstein \& McDaniel, 2010; Smith, 2010). Savine, McDaniel, Shelton, and Scullin (2012) recently demonstrated differences in how individuals temporally distribute attention to PM tasks. We asked a related but different question: Can we find direct evidence that participants process individual ongoing task items differently when they have PM requirements, as compared with when they do not? We answered this research question by 
examining the recognition of nontarget words previously presented in ongoing tasks with PM requirements.

There have been several theoretical proposals regarding the control processes allocated to PM tasks. According to the Smith (2003) preparatory attentional and memory processes (PAM) theory, individuals allocate "preparatory attention" to prepare for a switch between ongoing task and PM task responses. Similarly, Guynn (2003) proposed that individuals maintain their memory system in a retrieval mode, described as a mental set for treating ongoing task items as PM retrieval cues. Participants may also rehearse PM instructions (Smith, 2010). These generic PM processes can be distinguished from PM processes directed at individual ongoing task items. Guynn (2003) and the multiprocess view of PM (Einstein \& McDaniel, 2005) propose that participants periodically "check" or "monitor" for targets. In line with this, PAM proposes that preparatory attention serves the additional function of mapping individual ongoing task stimuli onto PM intentions (also see Savine et al., 2012; "attentional focus model").

Costs to nontarget ongoing task trials cannot be used to directly infer that individual ongoing task items have been differentially processed for PM, since these costs may also reflect generic PM processes. Findings of increased response time to PM lures demonstrate that ongoing task items can receive extra individual attention if their stimulus properties partially or exactly match PM targets (Knight, Meeks, Marsh, Cook, Brewer \& Hicks, 2011). PM goals are likely to be spontaneously retrieved when PM lures are processed, with the increased response time caused either by this initial noticing or by subsequent target verification, intention retrieval, or response coordination (Knight et al., 2011). In contrast, the present study is concerned with whether participants differentially process nontarget items that have not previously attracted attention because of their relationship to the conditions needed to satisfy the PM task. Participants performed a lexical decision task, either with or without PM requirements, followed by a surprise recognition memory task, where nontarget words from the lexical decision task were intermixed with new words. The recognition of nontargets previously presented in the ongoing task was expected to provide a measure of the differential processing of nontarget ongoing task items (Burt, Walker, Humphreys, \& Tehan, 1993; Humphreys, Maguire, McFarlane, Burt, Bolland, Murray \& Dunn, 2010).

Participants in the control condition performed the lexical decision task without a PM task. Some participants were required to make the $\mathrm{PM}$ response when presented with a specific word. Detecting a specific target is considered focal to lexical decision because lexical decision requires participants to decide whether letter strings are words or not. Other participants made the PM response when presented with a category exemplar. Detecting category exemplars is nonfocal because lexical decision does not require individuals to process the semantic features of letter strings necessary to make a category determination (Einstein \& McDaniel, 2005). PM performance is typically higher for specific (focal) than for categorical (nonfocal) targets (Marsh, Hicks, Cook, Hansen, \& Pallos, 2003).

We expected to replicate previous findings of costs to ongoing tasks for categorical (nonfocal) PM conditions, as compared with controls. Generic PM processes underlying these costs should not benefit recognition. In contrast, specific PM processes that operate on individual ongoing task items may improve recognition. Specifically, if participants in the nonfocal condition map the semantic features of letter strings onto the semantic features of their PM category, this elaboration in processing may increase the incidental learning of nontargets over and above that provided by making lexical decisions, enhancing the subsequent recognition of those nontargets (Craik \& Lockhart, 1972). There are typically no costs to lexical decisions for focal PM conditions (for an exception, see Smith, 2010). Numerous studies demonstrate that focal PM tasks can be spontaneously retrieved without the need to maintain PM cognitive control processes on nontarget trials (e.g., Harrison \& Einstein, 2010). We included a second focal condition where the importance of the PM task was emphasized (Loft \& Yeo, 2007). We expected that the focalimportance condition would show costs of similar size as the nonfocal condition due to the employment of a variety of possible general or specific cognitive control processes. However, there is still no logical necessity to retrieve semantic information in order to determine whether a letter string being processed corresponds to a specific target word. Thus, there is no basis to assume that the important focal PM task will enhance recognition. Inclusion of the focal-importance condition does, however, provide an experimental demonstration that the improved recognition of nontargets in the nonfocal condition is not an epiphenomenon of prolonged presentation (study) time during lexical decision. Finally, for completeness, a second nonfocal condition was included where the importance of the PM task was emphasized.

\section{Method}

\section{Participants}

Two hundred eighty-eight undergraduates participated and were randomly assigned to conditions. There were 96 participants in the control condition, and 48 participants in each of the four PM conditions. 


\section{Materials}

A pool of 384 medium frequency words (occurring 20-50 times per million) were randomly selected from the Sydney Morning Herald word database (Dennis, 1995). Of these, 184 were used as words, and 200 were converted to nonwords by randomly replacing each vowel in each word (e.g., chemist to chamust).

Two lists were created, and each list contained 92 words and 100 nonwords. Each list had a designated category of PM targets embedded within it (sports or fruits). Eight medium-typicality exemplars from each category were used as targets (Van Overschelde, Rawson, \& Dunlosky, 2004). In list A, the category was sport (golf, softball, soccer, cricket, hockey, lacrosse, tennis, rugby). In list B, the category was fruit (grape, pear, peach, melon, cherry, banana, plum, mango). Words and nonwords from one list were used as stimuli for lexical decision, and a random subset of nontarget words from the other list were used as new items in the recognition task. The assignment of lists to the lexical decision and recognition task was counterbalanced.

For the lexical decision task, the presentation of words and nonwords within each list was random, except that targets were presented in a random position between trials 5 and 25, 26 and 50, 51 and 75, 76 and 100, 101 and 125, 126 and 150,151 and 175, and 176 and 200. The focal conditions were presented with one category exemplar eight times, one at each allocated target trial position. Each exemplar of each category was used equally as often as a focal target across participants. In the nonfocal conditions, one category exemplar was presented in each allocated target trial position. Overall, in the nonfocal conditions, eight different category exemplars were presented. In the control condition, targets were also presented, but the targets held no special significance. Half the control condition we presented with one focal target, and half with category exemplars.

The recognition task presented 80 of the old words previously presented in the lexical decision task and 40 randomly selected new words from the alternative list. Old words were excluded from the recognition task if they were presented on the first two trials of the lexical decision task, were targets, or were presented in one of the two trials following targets. The recognition task consisted of four blocks of 30 words ( 20 old and 10 new). In order to approximately equate the average retention interval across items (Humphreys et al., 2010), the first 20 valid words presented in the lexical decision task were presented in the first recognition block, the next 20 lexical decision valid words were presented in the second block, and so on for the third and fourth blocks. The assignment of new words to the recognition task blocks was random, as was the presentation order of new and old words within each block.
Procedure

For lexical decision, participants were instructed to decide whether letter strings were English words or nonwords and to respond by pressing " $f$ " for a word or " $\mathrm{j}$ " for a nonword. The first display was a fixation point "+" displayed in white on a black background for $500 \mathrm{~ms}$. The fixation point was then replaced by a blank screen for $250 \mathrm{~ms}$, followed by the presentation of the letter string, which remained on the screen until the participant made a response.

Participants were told to respond as quickly and accurately as possible to the lexical decision task. Participants in the PM conditions were additionally instructed to press the "9" key when presented with a target during the ongoing task and were told that they could press the "9" key on one of the next two trials if they forgot to on the target trial. Participants in the focal conditions were instructed to press "9" whenever a specific target word was presented. Participants in the nonfocal conditions were instructed to press "9" when an exemplar of a target category was presented. The participants in the PM important conditions were additionally instructed that it was important that they remember to make the PM response. Participants completed a 3-min distractor puzzle before beginning lexical decision. Following the lexical decision task, participants completed a second 3-min distractor puzzle before performing the recognition task. On the recognition task, participants were presented with a 500-ms blank screen, followed by a single word. Participants pressed "o" if they believed that the word had been presented in the lexical decision task and " $n$ " if they believed that the word had not appeared in the lexical decision task.

\section{Results}

PM performance, response time to the lexical decision task, and hit rates, false alarm rates, and discrimination $\left(d^{\prime}\right)$ for the recognition task are presented in Table 1. First, we conducted 2 (focality) $\times 2$ (importance) between-subjects ANOVAs to confirm that the focality and importance manipulations affected PM and the ongoing task in a manner consistent with past research. We then conducted planned contrasts that directly paralleled our hypotheses (Rosenthal \& Rosnow, 1985), by comparing the ongoing task response time and recognition task performance for each experimental condition with those in the control condition.

Prospective memory

PM responses were scored as correct if participants pressed the "9" key on the target trial or on the following two trials. 
Table 1 Prospective memory accuracy (PM accuracy), lexical decision response time (LD RT), and hit rate, false alarm rate, and discrimination ( $d^{\prime}$ ) on the recognition task as a function of condition (with standard deviations in parentheses)

\begin{tabular}{|c|c|c|c|c|c|}
\hline & PM accuracy & LD RT & Hit & False alarm & $d^{\prime}$ \\
\hline Control $(N=96)$ & & $598(91)$ & $.67(.12)$ & $.26(.12)$ & $1.15(0.41)$ \\
\hline Focal standard $(N=48)$ & $.85(.20)$ & $621(85)$ & $.69(.11)$ & $.25(.12)$ & $1.26(0.53)$ \\
\hline Focal importance $(N=48)$ & $.94(.10)$ & $678(131)$ & $.70(.13)$ & $.27(.12)$ & $1.21(0.45)$ \\
\hline Nonfocal standard $(N=48)$ & $.60(.27)$ & $692(130)$ & $.77(.11)$ & $.28(.12)$ & $1.42(0.51)$ \\
\hline Nonfocal importance $(N=48)$ & $.77(.19)$ & 735 (137) & $.75(.12)$ & $.28(.13)$ & $1.35(0.42)$ \\
\hline
\end{tabular}

There were main effects of focality, $F(1,188)=50.60, p<.001$, $\eta_{\mathrm{p}}{ }^{2}=.21$, and importance, $F(1,188)=19.47, p<.001, \eta_{\mathrm{p}}{ }^{2}=$ .09 , but no interaction, $F(1,188)=1.59, p=.21, \eta_{\mathrm{p}}{ }^{2}=.01 . \mathrm{PM}$ accuracy was greater for the focal condition than for the nonfocal condition and was greater when PM importance was emphasized.

\section{Ongoing task}

Lexical decision accuracy was near ceiling $(M=.97)$ (all $\left.F_{\mathrm{s}}<1\right)$. For response times, we excluded the first two trials, target trials, PM false alarms, and the two trials following targets and false alarms. Only word trials were included, and we excluded incorrect lexical decisions and response times that were greater than $3 S D$ s from participants' grand mean. There were main effects of focality, $F(1,188)=13.12, p<.001, \eta_{\mathrm{p}}{ }^{2}=$ .07 , and importance, $F(1,188)=8.02, p=.01, \eta_{\mathrm{p}}{ }^{2}=.04$, but no interaction, $F<1$. Response times were greater for the nonfocal than for the focal condition and greater when PM importance was emphasized.

\section{Planned comparisons}

The summary of the literature by Smith et al. (2007) indicated that, if present, nonfocal and focal PM costs will be observed as large and medium size effects, respectively. The power to detect the large and medium size effects was .99 and .80, respectively (Faul, Erdfelder, Lang, \& Buchner, 2007). There was no a priori reason to expect a certain effect size for recognition, so we report the power to detect a medium size effect (.80). The parameter $d^{\prime}$ was used as the measure of discrimination. Old words were excluded from the recognition tasks analyses if they were incorrectly responded during the lexical decision task.

There was no cost for the focal-standard condition, as compared with the control condition as measured by lexical decision response time, $t(142)=1.47, p=.14, d=.26$. In addition, participants in the focal-standard condition $(M=$ .97) made more accurate lexical decisions than did the controls $(M=.96), t(142)=2.12, p=.04, d=.39$. On the recognition task, there were no differences in hit rate, $t(142)$
$=1.01, p=.31, d=.18$, false alarm, $t<1$, or discrimination, $t(142)=1.37, p=.17, d=.23$, between the focal-standard condition and controls.

Participants in the focal-important condition made slower lexical decisions than did the controls, $t(142)=4.31, p<.001$, $d=.72$. There was, however, a trend for participants in the focal-important condition $(M=.97)$ to make more accurate lexical decisions than did controls $(M=.96), t(142)=1.98, p=$ $.05, d=.37$. There was no correlation between lexical decision response time and $\mathrm{PM}$ accuracy, $r=.003, p=.98$, and no significant difference in response time to the four word trials preceding PM hits $(M=639 \mathrm{~ms})$, as compared with the four word trials preceding PM misses $(M=611 \mathrm{~ms}), t(16)=1.37$, $p=.19, d=.17$, indicating that the cost was not functional to PM. There were no differences in hit rate, $t(142)=1.27, p=$ $.21, d=.21$, false alarm, $t<1$, or discrimination, $t<1$, between the focal-important condition and controls.

Participants in the nonfocal-standard condition made slower lexical decisions than did controls, $t(142)=5.06, p<.001, d=$ .84 , with no difference in accuracy, $t(142)=1.62, p=.11, d=$ .29. Lexical decision response time was positively correlated with PM accuracy, $r=.37, p=.01$, and response times to the four word trials preceding PM hits $(M=692 \mathrm{~ms})$ were slower than those to the four word trials preceding PM misses $(M=$ $650 \mathrm{~ms}), t(39)=2.57, p=.01, d=.39$, both of which indicate that the cost was functional to PM. Participants in the nonfocalstandard condition had a higher hit rate, $t(142)=5.03, p<.001$, $d=.90$, and higher discrimination, $t(142)=3.36, p<.001, d=$ .57 , than did controls, with no difference in false alarm, $t<1$. For the nonfocal-standard condition, discrimination was positively correlated with PM accuracy, $r=.31, p=.03$. There was no difference in lexical decision response time for those words subsequently recognized $(M=698 \mathrm{~ms})$, as compared with those not recognized $(M=688 \mathrm{~ms}), t(47)=1.68, p=.32, d=.08$.

Participants in the nonfocal-important condition made slower lexical decisions than did controls, $t(142)=7.19$, $p<.001, d=1.2$, with no difference in accuracy, $t(142)=$ $1.70, p=.09, d=.31$. Lexical decision response time was positively correlated with PM accuracy, $r=.33, p=.02$, and response time to the four word trials preceding PM hits $(M=$ $737 \mathrm{~ms}$ ) were slower, as compared with the four word trials 
preceding PM misses ( $M=662 \mathrm{~ms}), t(37)=5.55, p<.001, d$ $=.54$, both of which indicate that the cost was functional to PM. Participants in the nonfocal-important condition had a higher hit rate, $t(142)=3.51, p<.001, d=.62$, and had higher discrimination, $t(142)=2.62, p=.01, d=.48$, than did controls, with no difference in false alarms, $t<1$. For the nonfocal-important condition, discrimination and PM accuracy were not correlated, $r=.12, p=.42$. There was no difference in lexical decision response time for those words that were subsequently recognized $(M=737 \mathrm{~ms})$, as compared with those not recognized $(M=728 \mathrm{~ms}), t(47)=1.04$, $p=.31, d=.06$.

\section{Discussion}

We have introduced a new dependent measure for examining whether participants differentially process individual nontarget items when they have PM requirements. Participants completed a lexical decision task with embedded PM requirements, followed by a surprise recognition test for nontarget ongoing task words. PM performance in both focal conditions was high. There were costs for the focal-important condition, but this cost was not functional to PM. It is likely that participants relied on spontaneous retrieval under focal conditions, without the need to maintain PM cognitive control processes on nontarget trials (Harrison \& Einstein, 2010). The cognitive control processes underlying costs in response to the PM importance manipulation would not have involved accessing semantic relationships and, thus, would not have benefited recognition. Costs to ongoing tasks were observed for both nonfocal conditions, and these costs were functional to PM. Both nonfocal conditions had an increased hit rate and increased discrimination on the recognition task, relative to controls. Furthermore, for the nonfocal standard condition, there was a positive correlation between discrimination and PM accuracy, suggesting that the improved recognition reflected processes employed during the ongoing task that were beneficial to PM.

We interpret these data as evidence that participants in the nonfocal conditions engaged specific PM processes to evaluate individual ongoing task items for their PM status. Specifically, participants in the nonfocal condition mapped the semantic features of letter strings onto the semantic features of their PM category. This elaboration in processing increased the incidental learning of nontargets and enhanced their subsequent recognition (Craik \& Lockhart, 1972). Our interpretation is in line with several PM theories that claim that individuals "check" or "monitor" for PM targets (Einstein \& McDaniel, 2005; Guynn, 2003; Savine et al., 2012; Smith, 2010). We are not claiming that generic PM processes play no role in PM retrieval. However, processes such as preparing to switch between ongoing task and PM task responses (Smith, 2010), or the instantiation of retrieval modes (Guynn, 2003), should not influence the incidental learning of individual nontargets or their subsequent recognition.

The costs for the focal-importance and the nonfocal standard conditions were of similar effect size. Thus, the lack of recognition benefit for the focal-importance condition indicates that it was the type of item-level processing required to detect nonfocal targets, rather than the prolonged study time, that underlies the nonfocal recognition benefit. In line with this, for the nonfocal conditions, lexical decision response times were not longer for words subsequently recognized, as compared with those not subsequently recognized. Costs to ongoing tasks potentially reflect both specific and generic PM processes. It is possible, then, that the semantic mapping of items to the PM category contributes to only a portion of the nonfocal PM costs, in which case we would not necessarily expect a strong withinsubjects relationship between the magnitude of costs and hit rates on the recognition test. Future research is needed to further examine the extent to which the cost to ongoing task measure and the recognition benefit measure we have introduced in the present article reflect similar underlying cognitive control processes.

The present data may appear at odds with the finding of Cook, Marsh, Clark-Foos, and Meeks (2007) that PM tasks imposed on participants when lists of words were studied decreased the subsequent recall of those words. Cook et al. concluded that PM task requirements during the study phase drew attention away from the creation of interitem associations that support free recall. In contrast, our recognition advantage depended on the incidental learning that occurred during the ongoing task when items were differentially processed for the PM task.

In conclusion, we have demonstrated that examining participants' recognition of nontarget words previously presented in ongoing tasks can be informative regarding understanding the processes underlying PM. Continuing to use different dependent measures should provide greater constraints for the various theoretical propositions presented in the event-based PM literature.

Author Note Shayne Loft, School of Psychology, The University of Western Australia. Michael S. Humphreys, School of Psychology, The University of Queensland.

This research was supported by Discovery Grant DP0986942 from the Australian Research Council awarded to Loft. We thank Louise Delane and Karli Riseborough for assistance in collecting and analyzing the data and Vanessa Bowden for commenting on the draft. 


\section{References}

Burt, J. S., Walker, M., Humphreys, M. S., \& Tehan, G. (1993). Associative priming in perceptual identification: Effects of prime processing requirements. Memory \& Cognition, 21, 125-137.

Cook, G. I., Marsh, R. L., Clark-Foos, A., \& Meeks, J. T. (2007). Learning is impaired by activated intentions. Psychonomic Bulletin and Review, 14, 101-106.

Craik, F. I. M., \& Lockhart, R. S. (1972). Levels of processing: A framework for memory research. Journal of Verbal Learning and Verbal Behavior, 11, 671-684.

Dennis, S. (1995). The Sydney Morning Herald word database. Noetica: Open Forum, 1(4). Retrieved from http://psy.uq.edu.au/CogPsych/ Noetica

Einstein, G. O., \& McDaniel, M. A. (1990). Normal aging and prospective memory. Journal of Experimental Psychology: Learning, Memory, and Cognition, 16, 717-726.

Einstein, G. O., \& McDaniel, M. A. (2005). Prospective memory: Multiple retrieval processes. Current Directions in Psychological Science, 14, 286-290.

Einstein, G. O., \& McDaniel, M. A. (2010). Prospective memory and what costs do not reveal about retrieval processes: A commentary on Smith, Hunt, McVay, and McConnell (2007). Journal of Experimental Psychology: Learning, Memory, and Cognition, 36, 1082-1088.

Faul, F., Erdfelder, E., Lang, A. G., \& Buchner, A. (2007). G*power 3: A flexible statistical power analysis program for the social, behavioural and biomedical science. Behavior Research Methods, 39, 175-191.

Guynn, M. J. (2003). A two-process model of strategic monitoring in event-based prospective memory: Activation/retrieval mode and checking. International Journal of Psychology, 38, 245-256.

Harrison, T. L., \& Einstein, G. O. (2010). Prospective memory: Are preparatory attentional processes necessary for a single focal cue? Memory and Cognition, 38, 860-867.

Humphreys, M. S., Maguire, A. M., McFarlane, K. A., Burt, J. S., Bolland, S. W., Murray, K. L., \& Dunn, R. (2010). Using maintenance rehearsal to explore recognition memory. Journal of
Experimental Psychology: Learning, Memory, and Cognition, $36,147-159$.

Knight, J. B., Meeks, J. T., Marsh, R. L., Cook, G. I., Brewer, G. A., \& Hicks, J. L. (2011). An observation of spontaneous noticing of prospective memory event-based cues. Journal of Experimental Psychology: Learning, Memory, and Cognition, 37, 298-307.

Loft, S., \& Yeo, G. (2007). An investigation into the resource requirements of event-based prospective memory. Memory and Cognition, 35, 263-274.

Marsh, R. L., Hicks, J. L., Cook, G. I., Hansen, J. S., \& Pallos, A. L. (2003). Interference to ongoing activities covaries with the characteristics of an event-based intention. Journal of Experimental Psychology: Learning, Memory, and Cognition, 29, 861-870.

Rosenthal, R., \& Rosnow, R. L. (1985). Contrast analysis: Focused comparisons in the analysis of variance. Cambridge, England: Cambridge University Press.

Savine, A. C., McDaniel, M. A., Shelton, J. T., \& Scullin, M. K. (2012). A characterization of individual differences in prospective memory monitoring using the complex ongoing serial task. Journal of Experimental Psychology. General, 141, $337-362$.

Smith, R. E. (2003). The cost of remembering to remember in eventbased prospective memory: Investigating the capacity demands of delayed intention performance. Journal of Experimental Psychology: Learning, Memory, and Cognition, 29, 347-361.

Smith, R. E. (2010). What costs do reveal and moving beyond the cost debate: Reply to Einstein and McDaniel. Journal of Experimental Psychology: Learning, Memory, and Cognition, 36, 1089-1095.

Smith, R. E., Hunt, R. R., McVay, J. C., \& McConnell, M. D. (2007). The cost of event-based prospective memory: Salient target events. Journal of Experimental Psychology: Learning, Memory, and Cognition, 33, 734-746.

Van Overschelde, J. P., Rawson, K. A., \& Dunlosky, J. (2004). Category norms: An updated and expanded version of the Battig and Montague (1969) norms. Journal of Memory and Language, 50, 289-335. 